\title{
Oculomotor interference during manual response preparation: evidence from the response-cueing paradigm
}

\author{
Lynn Huestegge • Jos J. Adam
}

Published online: 6 November 2010

(C) The Author(s) 2010. This article is published with open access at Springerlink.com

\begin{abstract}
Preparation provided by visual location cues is known to speed up behavior. However, the role of concurrent saccades in response to visual cues remains unclear. In this study, participants performed a spatial precueing task by pressing one of four response keys with one of four fingers (two of each hand) while eye movements were monitored. Prior to the stimulus, we presented a neutral cue (baseline), a hand cue (corresponding to left vs. right positions), or a finger cue (corresponding to inner vs. outer positions). Participants either remained fixated on a central fixation point or moved their eyes freely. The results demonstrated that saccades during the cueing interval altered the pattern of cueing effects. Finger cueing trials in which saccades were spatially incompatible (vs. compatible) with the subsequently required manual response exhibited slower manual RTs. We propose that interference between saccades and manual responses affects manual motor preparation.
\end{abstract}

Keywords Manual motor preparation - Finger precueing . Saccades $\cdot$ Eye movements $\cdot$ Cross-modal response interference

We thank Magali Kreutzfeldt for the collection of the data, and Jay Pratt, Shaun Vecera and two anonymous reviewers for helpful comments on earlier drafts of this paper.

L. Huestegge $(\bowtie)$

Institute of Psychology, RWTH Aachen University,

Jägerstrasse 17-19,

52066 Aachen, Germany

e-mail: lynn.huestegge@psych.rwth-aachen.de

J. J. Adam

Department of Movement Sciences, Maastricht University,

Maastricht, The Netherlands
Preparation of an action usually improves performance (Rosenbaum, 1980). In the finger-precueing task (Miller, 1982), participants respond to visuospatial stimuli with spatially corresponding responses from index and middle fingers of both hands placed adjacently. At the beginning of a trial, a warning stimulus (four horizontally arranged plus signs) indicates four possible stimulus locations. Then, a precue (two plus signs) of variable duration (preparation time) indicates two possible locations of the subsequent imperative stimulus (one plus sign). Thus, the precue transforms the original four-choice response task into a two-choice task.

Usually, four experimental conditions are distinguished. In the hand-cued condition, the precue corresponds to two fingers of the same hand (e.g., right index and middle finger). In the finger-cued condition, the precue corresponds to the same finger of different hands (e.g., left and right index finger). In the neither-cued condition, the precue corresponds to different fingers of different hands (e.g., left middle finger and right index finger). In a baseline condition the "precue" is identical to the warning stimulus, leaving the original four-choice task unaltered. The effectiveness of the precue is inferred from the response time (RT) advantage of the precue conditions relative to baseline (Hick, 1952; Hyman, 1953). When the preparation interval is below $1,500 \mathrm{~ms}$, RTs typically indicate that hand precues are most effective (i.e., the "hand advantage"), followed by finger and neither precues (e.g., Reeve \& Proctor, 1990).

Several explanations for the hand advantage have been proposed (Miller, 1982; Reeve \& Proctor, 1990), which have been incorporated in a compound, multi-factor model, the Grouping Model (Adam, Hommel, \& Umiltà, 2003, 2005; Adam \& Pratt, 2004, 2008). According to this model, 
Gestalt factors and inter-response dependencies mediate the formation of stimulus and response subgroups. Whereas hand cues (forming a perceptually and motorically strongly organized subgroup) are supposed to automatically activate the corresponding response subgroup, finger cues are assumed to involve slower, effortful processes to break up the automatically generated associations between stimulus and response subgroups.

An alternative interpretation of the hand advantage focuses on the idea that the spatial layout of the precues may cause shifts of visual attention and eye movements. Since it is well known that sudden on-/offsets of peripheral stimuli typically trigger saccades (e.g., Belopolsky \& Theeuwes, 2009; Findlay \& Walker, 1999; Yantis, 2005), it appears possible that eye movement responses may interfere with the manual responses. In this view, the unilateral hand precues would trigger saccades that spatially correspond to the required manual response. Finger precues, however, involve bilateral stimulation, so that saccades will equally likely be directed to either side, resulting in saccades that are spatially compatible or incompatible with the manual response. The influential premotor theory of attention (Rizzolatti, Riggio, Dascola \& Umilta, 1987) maintains that it is not possible to perform a saccade without a coordinate movement of spatial attention. Thus, whenever a saccade is directed in one direction and spatial attention (required to execute a manual response) in the opposite direction, performance deficits might arise. For example, recently we showed that the execution of spatially incompatible saccades and manual responses caused severe dual-task costs (Huestegge \& Koch, 2009).

The first aim of the present study is to test whether the probability of executing saccades during response preparation varies as a function of cue type. In particular, most saccades should occur in the hand-cued condition (due to unilateral precues), followed by the finger-cued condition (ambivalence of potential saccade directions due to bilateral precues) and the baseline condition (fewest saccades due to lack of display changes). As a second aim, we asked whether saccade execution modulates precueing effects by having participants either move their eyes freely or remain fixated. In particular, we explicitly test in the free viewing condition whether manual RTs in finger cueing trials are prolonged when spatially incompatible (vs. compatible) saccades occurred.

\section{Method}

Participants

Sixteen participants with normal or corrected-to-normal vision took part in the study (eight female and eight male, mean age $=24, S D=3.4$ ). They received money or course credits, and gave their informed consent.

Stimuli and apparatus

Participants were seated $67 \mathrm{~cm}$ in front of a 21 " cathode ray monitor (temporal resolution: $100 \mathrm{~Hz}$; spatial resolution: $1,240 \times 1,068$ pixels). The keyboard spacebar was used during calibration. Four keyboard keys served as response keys (Right-Ctrl, Right-Alt for the middle and index finger of the left hand, and Left-Arrow, RightArrow for the index and middle finger of the right hand, respectively). Saccades of the right eye were registered using a head-mounted Eyelink II infrared reflection system (SR Research, Canada) with a temporal resolution of $500 \mathrm{~Hz}$ and a spatial resolution $<0.022^{\circ}$. A chin rest minimized head movements.

A small green fixation point in the middle of the screen was present throughout the experiment. Stimuli were plus signs of $1^{\circ}$ visual angle each. The stimulus display subsequently consisted of a warning stimulus, a precueing stimulus, and an imperative stimulus, all of which were arranged horizontally on the central line of the monitor. While most finger-precueing studies utilize separate rows to present the three stimuli types, the present setup was also successfully implemented in previous research (see Moresi et al., 2008) and offers the advantage of a more accurate horizontal (as compared to vertical) eye movement measurement.

\section{Procedure and design}

Figure 1 depicts a schematic trial sequence. The warning stimulus was a row of four plus signs, separated by $4^{\circ}$. After $750 \mathrm{~ms}$, the precue (hand cue: the two left vs. right plus signs, finger cue: the two inner vs. outer plus signs) replaced the warning stimulus for either $100 \mathrm{~ms}$ or $500 \mathrm{~ms}$ (preparation interval, equally distributed across trials), followed by the imperative stimulus (one of the two plus signs from the precue), which remained for $2,000 \mathrm{~ms}$. The participants' task was to respond as quickly as possible to the position of the imperative stimulus by pressing the spatially corresponding key. In the baseline condition, all four plus signs remained present during the preparation interval. Finger cue, hand cue, and baseline conditions appeared equally often.

Each participant completed eight blocks of 30 trials each in a free viewing condition, in which participants were allowed to move their eyes freely. Another eight blocks of 30 trials each were completed in a remain-fixated condition, in which participants remained fixated on the small dot at the screen center. The sequence of fixation conditions was counterbalanced across participants. Within each block, 
Fig. 1 Schematic representation of a trial sequence (upper part: hand precueing condition, lower part: finger precueing condition). In the baseline condition (not depicted), the precue is identical to the warning stimulus

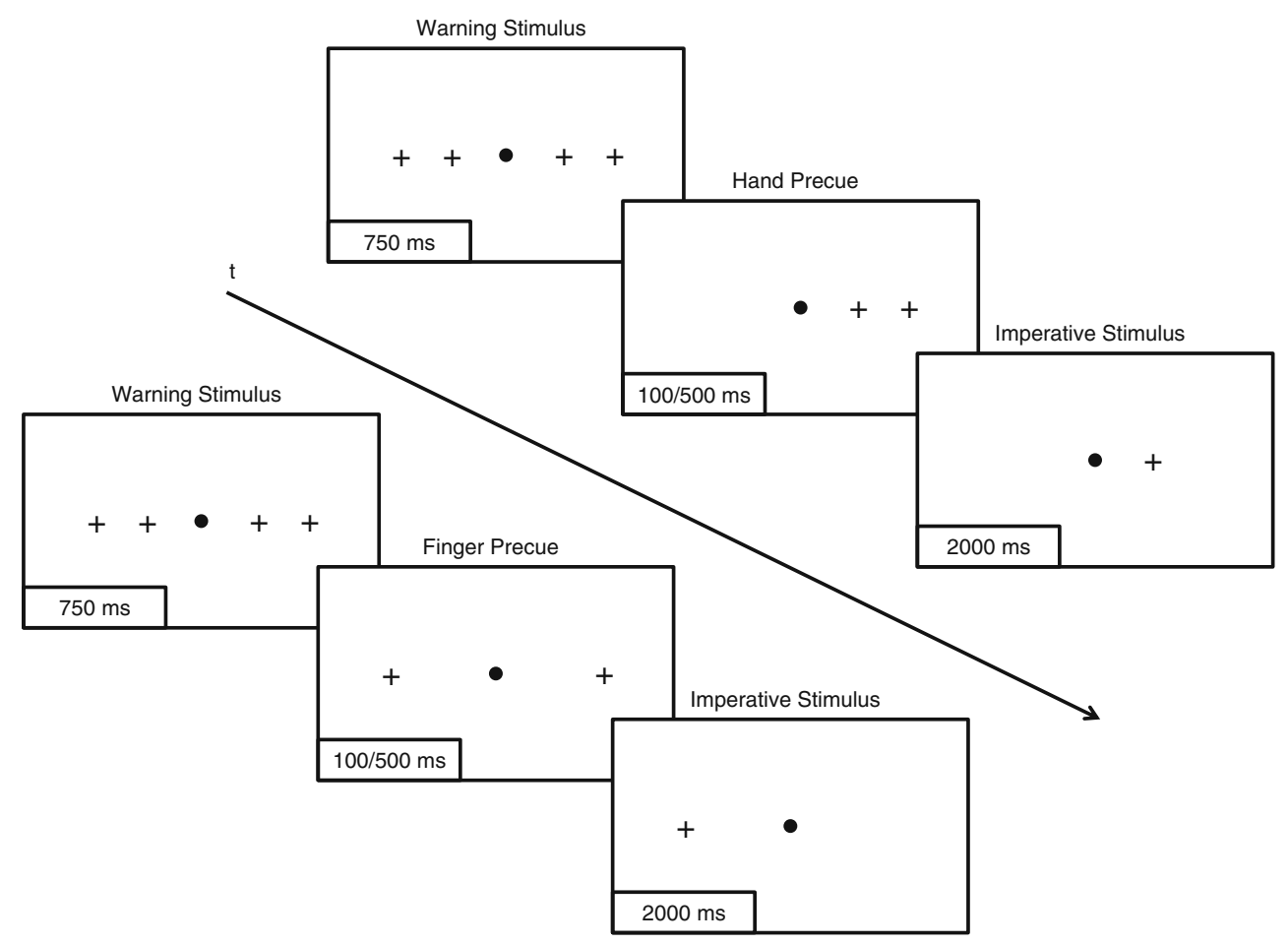

experimental trials were presented in randomized sequence. Prior to each block, participants underwent calibration. Prior to the experiment, 30 practice trials were administered.

The variables of cueing condition (hand-cued, finger-cued, baseline), preparation interval (100 ms vs. $500 \mathrm{~ms}$ ), and fixation condition (free vieweing vs. remain fixated) were manipulated intraindividually. We measured manual RTs, errors, and the occurrence of saccades (with a minimal amplitude of $>1.5^{\circ}$ ) during the cueing interval. To account for the delay between the point of no return of saccade initiation and the onset of each saccade (e.g., Findlay \& Walker, 1999), we did not include saccades within the first $50 \mathrm{~ms}$ during the preparation interval, but included saccades initiated up to $50 \mathrm{~ms}$ after the termination of the preparation interval.

\section{Results}

In the remain-fixated condition erroneous saccades (with an amplitude of $>1^{\circ}$ ) occurred in $5.8 \%$ of trials. These trials were excluded from further analyses.

Overall manual RT effects Figure 2 depicts mean RTs. A three-way ANOVA revealed a significant effect of cueing condition, $F(2,30)=145.79, p<.001, \eta_{p}^{2}=.91$. Mean RTs amounted to $477 \mathrm{~ms}(S E=16)$ in the hand-cued condition, $498 \mathrm{~ms}(S E=16)$ in the finger-cued condition, and $556 \mathrm{~ms}(S E=14)$ in the baseline condition. There was also a significant effect of preparation interval, $F(1,15)=$
121.65, $p<.001, \eta_{p}{ }^{2}=.89$, indicating faster RTs for longer preparation intervals ( $485 \mathrm{~ms}, S E=16$ ) as compared to shorter preparation intervals ( $535 \mathrm{~ms}, S E=15)$. There was no significant effect of fixation condition, $F(1,15)=2.61$, $p=.127$.

Most importantly, there was a significant interaction of cueing condition and fixation condition, $F(2,30)=3.67$, $p=.037, \eta_{p}^{2}=.20$, indicating that the occurrence of saccades during the cueing interval significantly altered the size of the precueing effects. There was a significant interaction of preparation interval and cueing condition,

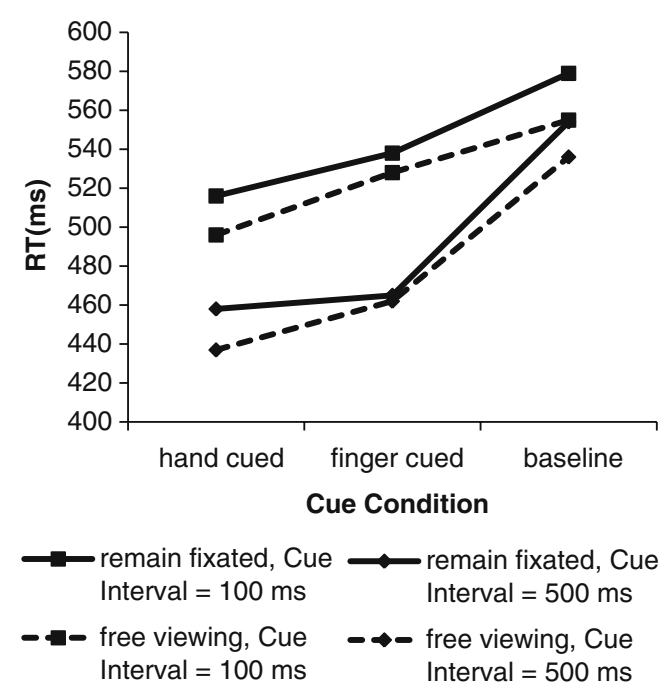

Fig. 2 Response times (RTs) as a function of cue condition, preparation (cue) interval, and fixation condition 
$F(2,30)=23.83, p<.001, \eta_{p}^{2}=.61$, indicating greater precueing benefits (for the hand- and finger-cued conditions relative to the baseline condition) for the 500-ms than for the 100 -ms preparation interval. There was no significant interaction of fixation condition and cueing interval, and no significant three-way interaction, both $F<1$.

Effects of fixation condition on hand and finger cueing effects To further qualify the interaction between cueing condition and fixation condition, we conducted two separate three-way ANOVAs. The first ANOVA differed from the above-reported ANOVA in that the cueing condition variable only included the "hand cued" and "baseline" conditions. The corresponding interaction of fixation condition and cueing condition was not significant, $F<1$. The second ANOVA only included the "finger cued" and "baseline" condition, and resulted in a significant interaction of fixation condition and cueing condition, $F(1,15)=6.85, p=.019, \eta_{p}^{2}=.31$. Taken together, these analyses indicate that only the finger cueing advantage (relative to baseline) was modulated by fixation condition, whereas the hand cueing advantage remained unaffected.

A closer inspection of the remain fixated condition showed that RTs for hand cueing and finger cueing were not significantly different in the 500 -ms cueing interval ( $458 \mathrm{~ms}$ vs. $465 \mathrm{~ms}, t<1$ ), but were so in the $100-\mathrm{ms}$ cueing interval ( $516 \mathrm{~ms}$ vs. $538 \mathrm{~ms}, t(15)=4.25, p=.001)$, indicating that the suppression of saccades did not fully eliminate RT differences between hand cues and finger cues.

Manual errors The overall mean manual error rate amounted to $2.6 \%$, which was deemed too low to permit meaningful statistical analyses. Participants made the most errors in the finger-cued condition (3.9\%), fewest in the hand-cued condition (1.8\%), and an intermediate percentage of errors in the baseline condition (2.2\%). Manual error rates across fixation conditions were virtually identical $(2.6 \%$ for free viewing vs. $2.7 \%$ for remain fixated), as were manual error rates for the different preparation intervals $(2.6 \%$ for $100-\mathrm{ms}$ vs. $2.8 \%$ for $500-\mathrm{ms}$ preparation interval).

Eye movement analyses The occurrence of eye movements was further analyzed during free viewing. A two-way ANOVA resulted in a significant main effect of cueing condition, $F(2,30)=39.13, p<.001, \eta_{p}^{2}=.72$. More specifically, saccades occurred in $35.5 \%(S E=2.9)$ of trials in the hand-cued condition, in $24.8 \%(S E=3.6)$ of trials in the finger-cued condition, and in $15.6 \%(S E=2.9)$ of trials in the baseline condition. There was also a significant main effect of preparation interval, $F(1,15)=96.53, p<.001$, $\eta_{p}^{2}=.87$, indicating more saccades during the longer cueing interval (44.2\%) compared to the shorter cueing interval (6.4\%), and a significant two-way interaction, $F(2,30)=46.17, p<.001, \eta_{p}^{2}=.76$, indicating a stronger effect of cueing condition for the 500-ms preparation interval compared to the $100-\mathrm{ms}$ preparation interval. Post-hoc $t$-tests revealed that for the $500-\mathrm{ms}$ condition, there was a significant difference between the hand-cued $(64.5 \%)$ and finger-cued $(43.1 \%)$ conditions, $t(15)=4.91$, $p<.001$, and between the finger-cued and baseline $(25.0 \%)$ conditions, $t(15)=4.70, p<.001$, whereas there were no corresponding differences in the 100 -ms condition (overall $M=6.4 \%$; both $t<1$ ), probably due to the comparatively rare occurrence of saccades.

Effects of cross-modal response compatibility A final analysis tested whether the direction of the initial saccade during the preparation interval affected RTs. For this analysis, we only used the finger-cued condition, where visual changes occurred in both hemifields. We restricted our analysis to the $500-\mathrm{ms}$ preparation interval condition, which contained a sufficiently large number of saccades. Importantly, trials in which saccade direction and subsequent key location were compatible yielded faster RTs than incompatible trials ( $442 \mathrm{~ms}, S E=72$ vs. $475 \mathrm{~ms}, S E=76$ ), $t(15)=2.18, p=.046$, see Fig. 3). This key finding indicates that saccade direction during preparation in the finger-cued condition significantly affected subsequent manual RTs. Furthermore, finger-cued trials with compatible eye movements generated RTs that were similar to those generated by hand cues (i.e., $442 \mathrm{~ms}$ vs. $437 \mathrm{~ms}$, $t<1$ ), whereas finger-cued trials with incompatible saccades generated significantly longer RTs than those generated by hand cues (i.e., $475 \mathrm{~ms}$ vs. $437 \mathrm{~ms}, t(15)=2.42, p=.029$ ).

In hand-cued conditions saccades were usually directed towards the center between the two cueing stimuli (known

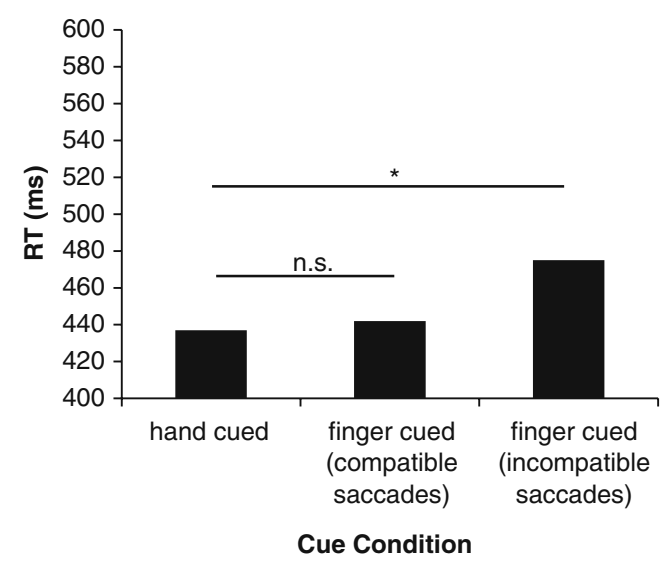

Fig. 3 Response times (RTs) as a function of the spatial compatibility of the required manual response (left vs. right) and the saccade direction (left vs. right) during the preparation interval in finger-cued trials (free viewing, $\mathrm{SOA}=500 \mathrm{~ms}$ ). The leftmost column presents the corresponding hand-cued RTs 
as the "global effect," see Findlay \& Walker, 1999). Hence, here it was not possible to analyze effects of the correspondence between the fixated cueing stimulus and the imperative stimulus.

\section{Discussion}

The occurrence of saccades covaried with cue condition. When the time window was large enough for saccades to occur (500-ms preparation interval), we found the fewest saccades in the baseline condition (due to the lack of a display change), more saccades in the finger-cued condition, and the most saccades in the hand-cued condition. This pattern was expected, since hand cues unambiguously attract attention towards one single hemifield, whereas finger cues involve a display change in both hemifields, probably attenuating the occurrence of saccades.

Importantly, the comparison of "free viewing" and "remain fixated" conditions revealed the key finding of this study, namely that the occurrence of saccades modulated precueing effects only in the finger cue condition. Given that the hand cue condition elicited most saccades, one might have expected an effect of fixation condition also on hand cues. However, this was not the case, indicating that the mere presence of saccades does not per se affect manual motor preparation. Instead, adverse effects appear to be specifically related to the spatial (in)congruency between saccades and manual responses.

The crucial role of spatial (in)congruency between saccades and manual responses was evident in the fingercueing condition during free viewing. Since participants could not know in advance where the imperative stimulus would appear, about half of the saccades during the preparation interval were directed into the opposite direction relative to the subsequent imperative stimulus (and corresponding manual response). These trials with spatially incompatible saccades substantially slowed down manual RTs (i.e., a robust interference effect of $33 \mathrm{~ms}$ ), whereas RTs in compatible trials did not significantly differ from hand-cueing conditions. Thus, the advantage of hand cues (vs. finger cues) crucially seems to depend on cross-modal response interference, a finding that is well in line with previous research showing that spatially incompatible saccades and manual responses lead to considerable interference (Huestegge \& Koch, 2009, 2010a).

Interestingly, with the $100-\mathrm{ms}$ preparation interval, there still was a RT difference between finger cues and hand cues in the remain-fixated condition, indicating that the suppression of saccades did not fully eliminate RT differences. However, it is possible that this remaining difference can be explained as an effect of some trials in which visual attention was covertly shifted into an incompatible direction relative to the manual response.

This explanation, as well as the overall data pattern, is by and large compatible with the premotor theory of attention (Rizzolatti et al., 1987; Rizzolatti, Riggio \& Sheliga, 1994), which maintains that covert spatial attention derives from the activation of brain maps engaged in sensorimotor transformation processes. More specifically, this activation is assumed to result in both an increase in the readiness to execute a motor response (e.g., a manual response) at a specific location and a facilitation of perceptual processing (allocation of covert and overt attention) at that location (see Huestegge \& Koch, 2010b). Importantly, motor responses and spatial attention here are not exclusively linked to the visual system, an assumption that is supported by empirical evidence for a spatial coupling of perception and action in other modalities, such as visual/auditory attention and manual movements (e.g., Castiello, 1996; Craighero, Fadiga, Rizzolatti, \& Umiltà, 1999; Deubel, Schneider, \& Paprotta, 1998; Eimer, van Velzen, Gherri, \& Press, 2006; Tipper, Lortie, \& Bayliss, 1992; Spence \& Driver, 1996). Thus, this framework provides a mechanism to explain why in the finger-cued condition it appears difficult to attend to one direction (during the preparation interval) while preparing a manual response in the opposite direction.

Taken together, the results suggest that beside stimulus and response grouping processes and corresponding mapping mechanisms (Adam et al., 2003, 2005), cross-modal response interference between oculomotor and manual responses is another important factor that needs to be considered for explaining the hand cue advantage and manual motor preparation in general. However, the relative weight of these different explanatory sources can only be assessed in future research where the present manipulations are systematically combined with variables that affect perceptual and motor-based grouping processes. Finally, the study underlines the importance of eye movement analyses in manual response paradigms that involve spatially distributed visual stimulation (see also Buetti \& Kerzel, 2010).

Open Access This article is distributed under the terms of the Creative Commons Attribution Noncommercial License which permits any noncommercial use, distribution, and reproduction in any medium, provided the original author(s) and source are credited.

\section{References}

Adam, J. J., Hommel, B., \& Umiltà, C. (2003). Preparing for perception and action (I): The role of grouping in the responsecuing paradigm. Cognitive Psychology, 46, 302-358. 
Adam, J. J., Hommel, B., \& Umiltà, C. (2005). Preparing for perception and action (II): Automatic and controlled processes in response cuing. Visual Cognition, 12, 1444-1473.

Adam, J. J., \& Pratt, J. (2004). Dissociating visual attention and effector selection in spatial precuing tasks. Journal of Experimental Psychology: Human Perception and Performance, 30, 1092-1106.

Adam, J. J., \& Pratt, J. (2008). Motor set modulates automatic priming effects of uninformative cues. Acta Psychologica, 128, 216-224.

Belopolsky, A. V., \& Theeuwes, J. (2009). When are attention and saccade preparation dissociated? Psychological Science, 20, $1340-1347$.

Buetti, S., \& Kerzel, D. (2010). Effects of saccades and response type on the Simon effect: If you look at the stimulus, the Simon effect may be gone. The Quarterly Journal of Experimental Psychology, 63, 2172-1289.

Castiello, U. (1996). Grasping a fruit: Selection for action. Journal of Experimental Psychology: Human Perception and Performance, 22, 582-603.

Craighero, L., Fadiga, L., Rizzolatti, G., \& Umiltà, C. (1999). Action for perception: A motor-visual attentional effect. Journal of Experimental Psychology: Human Perception and Performance, $25,1673-1692$.

Deubel, H., Schneider, W. X., \& Paprotta, I. (1998). Selective dorsal and ventral processing: Evidence for a common attentional mechanism in reaching and perception. Visual Cognition, 5, 81-107.

Eimer, M., van Velzen, J., Gherri, E., \& Press, C. (2006). Manual response preparation and saccade programming are linked to attention shifts: ERP evidence for covert attentional orienting and spatially specific modulations of visual processing. Brain Research, 1105, 7-19.

Findlay, J. M., \& Walker, R. (1999). A model of saccade generation based on parallel processing and competitive inhibition. The Behavioral and Brain Sciences, 22, 661-674.

Hick, W. E. (1952). On the rate of gain of information. Quarterly Journal of Experimental Psychology, 4, 11-26.

Huestegge, L., \& Koch, I. (2009). Crosstalk between simultaneously executed saccades and manual responses. Journal of Experimen- tal Psychology: Human Perception and Performance, 35, 352362.

Huestegge, L., \& Koch, I. (2010a). Crossmodal action selection: Evidence from dual-task compatibility. Memory \& Cognition, 38, 493-501.

Huestegge, L., \& Koch, I. (2010b). Fixation disengagement enhances peripheral perceptual processing: Evidence for a perceptual gap effect. Experimental Brain Research, 201, 631-640.

Hyman, R. (1953). Stimulus information as a determinant of reaction time. Journal of Experimental Psychology, 45, 188-196.

Miller, J. (1982). Discrete versus continuous models of human information processing: In search of partial output. Journal of Experimental Psychology: Human Perception and Performance, 8, 273-296.

Moresi, S., Adam, J. J., Rijcken, J., Van Gerven, P. W. M., Kuipers, H., \& Jolles, J. (2008). Pupil dilation in response preparation. International Journal of Psychophysiology, 67, 124-130.

Reeve, T. G., \& Proctor, R. W. (1990). The salient-features coding principle for spatial- and symbolic-compatibility effects. In R. W. Proctor \& T. G. Reeve (Eds.), Stimulus-response compatibility (pp. 163-180). Amsterdam: North-Holland.

Rizzolatti, G., Riggio, L., Dascola, I., \& Umilta, C. (1987). Reorienting attention across the horizontal and vertical meridians: evidence in favor of a premotor theory of attention. Neuropsychologia, 25, 31-40.

Rizzolatti, G., Riggio, L., \& Sheliga, B. M. (1994). Space and selective attention. In C. Umilta \& M. Moscovitch (Eds.), Attention and performance $X V$ (pp. 231-265). Cambridge: MIT Press.

Rosenbaum, D. A. (1980). Human movement initiation: specification of arm, direction, and extent. Journal of Experimental Psychology: General, 109, 444-474.

Spence, C., \& Driver, J. (1996). Audiovisual links in endogenous covert spatial attention. Journal of Experimental Psychology: Human Perception and Performance, 22, 1005-1030.

Tipper, S. P., Lortie, C., \& Bayliss, G. C. (1992). Selective reaching: evidence for action centered attention. Journal of Experimental Psychology: Human Perception and Performance, 18, 891-905.

Yantis, S. (2005). How salient stimuli win the battle for awareness. Nature Neuroscience, 8, 975-977. 